\title{
Substituting management for cash inputs in organic systems
}

\author{
Jim Bender
}

The common assertion that organic farming replaces some cash inputs with management is sometimes taken as an indirect way of saying that it requires more labor as well. This is an unfortunate assumption because it makes organic systems seem less appealing. True, organic farming systems do replace some cash inputs with labor. However, they are impressive in the way they replace some of the inputs of conventional systems with pure management.

I will illustrate this important point with an example from each of the four seasons, plus a more general contrast between organic and conventional systems. The examples compare a typical diversified organic farm to a conventional corn-soybean system. The idea underlying all these examples is that, while achieving a particular goal in a conventional system might require direct expenditure of cash or labor, this same goal might be achieved on an organic farm as an additional consequence of a method chosen for other reasons, to achieve goals common to both systems.

Spring: Soil compaction

Soil compaction is a pervasive problem in contemporary agriculture. The main technique to deal with it in conventional systems is deep tillage with implements designed largely, or exclusively, for that purpose, such as chisel plows and subsoilers. The required labor and machinery are significant cash costs.

In contrast, a typical organic farm can use a very different, indirect strategy. This system will include legumes in its rotation for reasons that typically include fixing nitrogen, providing feed or ground cover, bringing trace minerals up from the subsoil, increasing the number of earthworms, and improving soil tilth. These are sufficient reasons for raising legumes. However, their deep, penetrating roots also help reduce the problem of soil compaction. Soil scientists disagree on just how much, and for how long, legumes reduce compaction compared to tillage. But both clearly help in different ways. Because the labor and expense of growing legumes is already justified by the benefits mentioned earlier, their contribution to ameliorating soil compaction can be regarded as free.

Jim.

Jim Bender is an organic farmer near Weeping Water,

\section{Summer: Weed control}

The problems of weed control vary with the season, as do the opportunities for dealing with them. A serious limitation of conventional chemical weed control is that it requires a substantial commitment of money and effort at the beginning of the growing season. This precludes cutting costs by adjusting to actual conditions later in the season.

A good organic sytem will maximize its flexibility in responding to changing conditions through staggered planting. In growing soybeans, for example, a prudent organic farmer will not follow the conventional practice of planting the entire crop as quickly as possible, but in a sense will plant it as slowly as possible. The total soybean acreage will be planted in much smaller units, with as much as several days allowed to pass between when successive units are planted. This allows the organic farmer to be in the best position to cope with a wet season or to take advantage of a dry one. In a wet season different fields will have different conditions; there will be more time to tend to fields with cultural methods because of the pauses between plantings. Because of the staggered planting, the farmer will have time to give extra attention to weed control if wet conditions make weed problems especially serious. Also in a wet season, because of the staggered planting, only some fields will have the severest weed problem; the farmer can give these fields extra attention. In a dry season, when weed problems are not so severe, the farmer can do as little as is necessary to control weeds, perhaps only one cultivation, with no rotary hoeing or harrowing.

There are two important points to be made here. First, the organic farmer's strategy for coping with the uncertainties of weed control arises automatically from the staggered planting. It does not cost more, it requires virtually no additional labor, and often will improve yields. Second, in dry years, when weed control is less of a problem, the organic farmer is in a better position to take advantage of this situation, to avoid some of the weed control costs to which the conventional system is already committed. Again, this comes from the management system, not from additional cash expenses or labor, in contrast to the chemical weed control methods used on conventional farms.

continued on page 46 


\section{OPINION-continued from page 2}

\section{Fall: Harvest and grain handling}

On a conventional corn-soybean farm, all crops are harvested in the fall. Here, good harvest management means timeliness. Timeliness, on medium to large farms, entails large, modern equipment to harvest, move, and condition the crop, plus considerable storage capacity and perhaps extra labor.

Contrast the capital requirements of this approach to a typical organic farm, again of medium to large size. Because of the more diversified crop mix, a substantial portion of the cropland is in pasture, legumes, and crops harvested in the summer. This reduces the pressure for timeliness in the fall and, therefore, also reduces the need for expensive harvest equipment. The decision to diversify, therefore, by itself reduces costs. Of course, this saving is partly offset by the organic farmer's hay handling equipment. However, reducing the size of a combine by just one size can by itself save more than the cost of a hay bailer.

Winter: Preparing harvested row crop fields for the next year's crop

Organic farmers are not the only ones who raise livestock. However, livestock are declining on conventional farms, whereas they are generally considered indispensable on organic farms. Therefore, the role of livestock provides an important area of contrast.

Preparing a field for the following year's crop includes preventing volunteer plants, managing crop residues, and providing adequate fertility. The conventional farmer typically addresses the first of these with herbicides, the second by residue shredders and tillage, and the third with purchased fertilizers. Each imposes cash costs.

In contrast, assume that the organic farmer has beef cattle grazing harvested row crop fields and that these cattle provide adequate financial return to justify their inclusion in the farming system. By grazing the harvested row crop fields, the cattle also are addressing each of the requirements for preparing for the next crop. They reduce volunteering by consuming the grain that causes the problem. They reduce the tillage requirement by consuming some of the residues. Finally, they contribute to fertility by converting the residue to manure. Once again, therefore, the organic farmer partially avoids some of the cash inputs or labor requirements of a conventional system because of a management decision that had another objective but provides other benefits in addition.

General contrast between systems: Risk management

Risk management is a central concern in evaluating competing farming systems. A conventional corn-soybean system is notoriously vulnerable to uncertainties. A farmer who is not willing to accept the risk must purchase crop insurance. Either the risk itself, or the insurance needed to avoid it, is part of the cost of production.

An organic farm, in contrast, reduces risk through diversification. Drought, for example, generally begins to threaten crops in summer. While a corn-soybean farmer is very vulnerable, on a typical organic farm one or two crops of hay have already been harvested, wheat and oats are ready for harvest, and livestock have grazed the available forage. Although this system does not avoid risk entirely, it will fare better than its conventional counterpart under severe drought. Once again, if there already were sufficient reasons to diversify, the reduction in risk is an additional benefit of a management decision.

Conclusion

I have offered five illustrations spanning the seasons and covering several basic features of a farming system, of how organic farms may eliminate some of the cash inputs of conventional agriculture not by additional labor but by how the system is managed. Indeed, in several examples, the management of organic systems replaces even the labor of a conventional system. In evaluating these competing systems, it is important to recognize the distinct way that organic systems can often solve their problems, that is, by using techniques that automatically serve additional purposes along with the primary objectives that by themselves have already covered the costs.

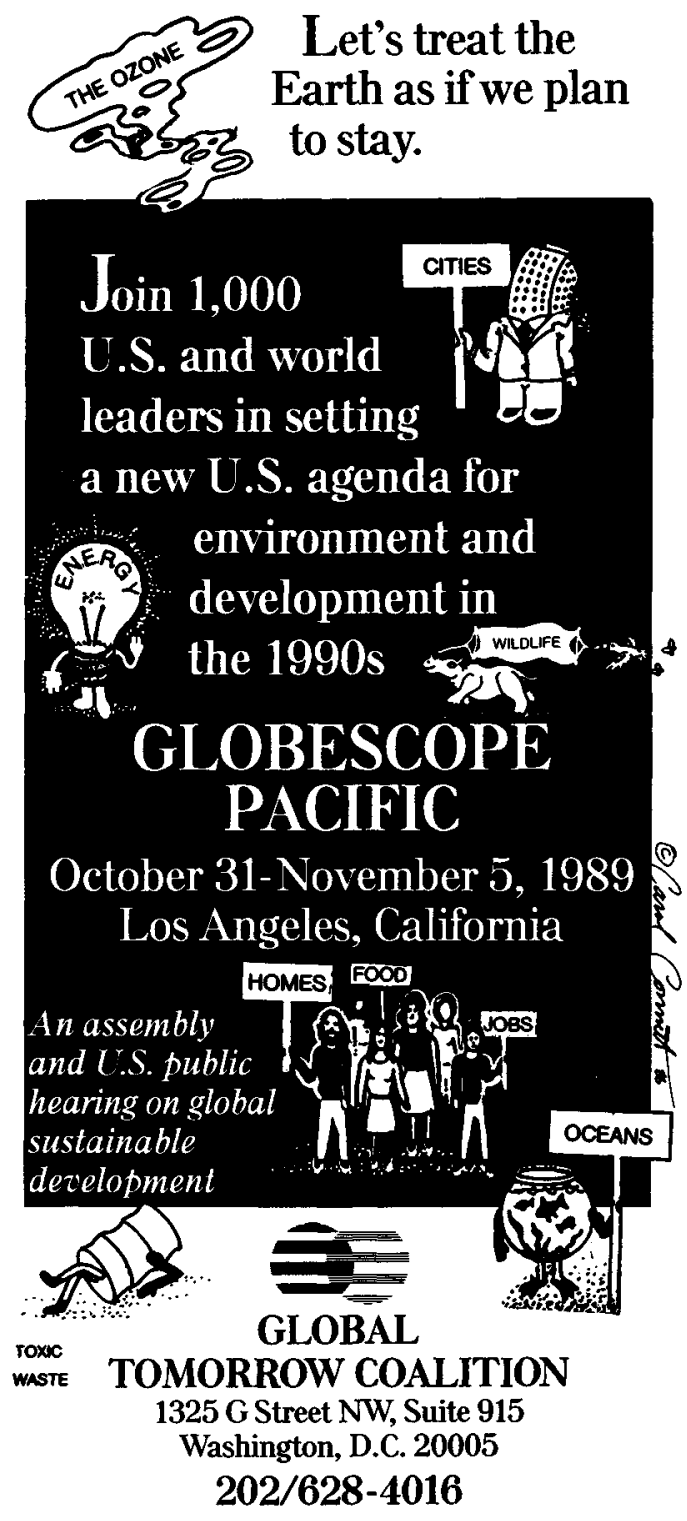

American Journal of Alternative Agriculture 